IMSc-97/11/39

\title{
Quantum Black Hole Entropy
}

\author{
Romesh K. Kaul and Parthasarathi Majumdar* \\ The Institute of Mathematical Sciences, CIT Campus, Madras 600113, India.
}

\begin{abstract}
We derive an exact formula for the dimensionality of the Hilbert space of the boundary states of $S U(2)$ Chern-Simons theory, which, according to the recent work of Ashtekar et al, leads to the Bekenstein-Hawking entropy of a four dimensional Schwarzschild black hole. Our result stems from the relation between the (boundary) Hilbert space of the Chern-Simons theory with the space of conformal blocks of the Wess-Zumino model on the boundary 2sphere.
\end{abstract}

The issue of the Bekenstein-Hawking (B-H) [1], [2] entropy of black holes has been under intensive scrutiny for the last couple of years, following the derivation of the entropy of certain extremal charged black hole solutions of toroidally compactified heterotic string and also type IIB superstring from the underlying string theories [3], [4]. In the former case of the heterotic string, the entropy was shown to be proportional to the area of the 'stretched' horizon of the corresponding extremal black hole, while in the latter case it turned out to be precisely the B-H result. The latter result was soon generalized to a large number of four and five dimensional black holes of type II string theory and M-theory (see [5] for a review), all of which could be realized as certain D-brane configurations and hence saturated the BPS bound. Unfortunately, the simplest black hole of all, the four

*email: kaul, partha, @imsc.ernet.in 
dimensional Schwarzschild black hole, does not appear to be describable in terms of BPS saturating D-brane configurations, and hence is not seemingly amenable to such a simple analysis.円 Results for near-extremal black holes, modeled as near-BPS states of string and M-theory, have also been obtained [5], pertaining both to their entropy and also Hawking radiation. In the majority of the cases considered, complicated (sometimes intersecting) configurations of D-branes were treated in the 'effective string approximation' [6]; in this approximation, computations effectively reduce to that in a two dimensional conformal field theory [7].

The B-H entropy of a four dimensional Schwarzschild black hole has been obtained, for large areas of the event horizon, within the alternative framework of canonical quantum gravity [8] by Krasnov and Ashtekar et al [9], [10], up to an overall constant of $O(1)$ known as the Immirzi parameter [11] (which essentially characterizes inherent ambiguities in the quantization scheme, and is therefore present in the quantum theory even in the absence of black holes). The black hole spacetime is considered as a 4-fold bounded by the surface at asymptotic null infinity (on which standard asymptotically flat boundary conditions hold) and the event horizon (on which boundary conditions, special to the spherically symmetric Schwarzschild geometry are assumed). The action embodying the assumed boundary conditions consists of, over and above the Einstein-Hilbert action (in the Ashtekar variables [8]), an $S U(2)$ Chern-Simons (CS) gauge theory 'living' on a coordinate chart of constant finite cross sectional area $A_{S}$ (and possessing some other properties) on the horizon. The ChernSimons coupling parameter $k \sim A_{S}$. In the Hamiltonian formulation of the theory, the boundary conditions are implemented as a condition on the phase space variables restricted to the boundary (2-sphere) of a spacelike 3-surface intersecting the constant area coordi-

\footnotetext{
${ }^{1}$ Recently, Sfetsos and Skenderis, hep-th/9711138, have obtained the B-H result for the $4 \mathrm{~d}$ Schwarzschild black hole by a U-duality map to the 3d BTZ black hole whose entropy has been calculated, again for large areas, by Carlip [12]. See also, Arguiro et al, hep-th/9801053.
} 
nate patch on the horizon. This results in a reducible connection variable which is gauge fixed (on the boundary) to the $U(1)$ subgroup of the $S U(2)$ invariance of the CS theory. In the quantum theory, the boundary conditions, implemented as an operator equation, imply that the space of surface (boundary) quantum states is composed of subspaces given by the Hilbert space of the $U(1)$ CS theory, on the boundary 2-sphere with finitely many punctures p labeled by spins $j_{p}$. Now, it has been argued [12] that boundary (or 'edge') states play the major role in producing black hole entropy. Likewise, the entropy of the black hole under consideration is assumed to emerge only from the surface states, and defined by tracing over the 'volume' states. It is then given by $S_{b h}=\ln N_{b h}$ where $N_{b h}$ is the number of boundary CS states. This number is next obtained from the dimensionality of the Hilbert space of boundary $U(1)$ CS states on the punctured 2-sphere. Finally, this is compared with the spectrum [13] of the area operator [14] in canonical quantum gravity, known, upto the Immirzi parameter, in terms of spins $j_{p}$ on the punctures $p$. For large number of punctures and large area, it is seen to be proportional to the logarithm of the dimensionality of the space of boundary CS states (i.e., the entropy). A particular choice of the Immirzi parameter then reproduces the Bekenstein Hawking value.

Recall that the B-H entropy of a black hole was proposed on the basis of semiclassical analyses, and as such, is by no means beyond modification in the full quantum theory. One of the simplifying steps in the above derivation was the reduction of the gauge group of the CS theory from the original $S U(2)$ to $U(1)$, by gauge fixing the connection on the boundary 2-sphere. As admitted by the authors, this is not a necessary step. Indeed, there exist powerful results relating the state space of CS theories on 3-folds with boundary to the conformal blocks of an $S U(2)$ Wess-Zumino model of level $k$ on that boundary 115 . It stands to reason that the entropy, derived from such considerations, will be more exact quantum mechanically. In this paper, we focus on such a calculation of the entropy, using the formalism of two dimensional conformal field theory.

More specifically, we compute the number of conformal blocks of an $S U(2)_{k}$ Wess-Zumino theory on a punctured 2 -sphere, for a set of punctures $\mathcal{P} \equiv\{1,2, \ldots, p\}$ where these punc- 
tures are labeled by the spin $j_{p}$, for arbitrary level $k$ (corresponding to an arbitrary area of the cross section of the patch chosen on the horizon). This number can be computed in terms of the so-called fusion matrices $N_{i j}{ }^{r}[16]$

$$
N^{\mathcal{P}}=\sum_{\left\{r_{i}\right\}} N_{j_{1} j_{2}}^{{ }^{r_{1}}} N_{r_{1} j_{3}}^{r_{2}} N_{r_{2} j_{4}}^{r_{3}} \ldots \ldots N_{r_{p-2} j_{p-1}}^{j_{p}}
$$

Here, each matrix element $N_{i j}{ }^{r}$ is 1 or 0 , depending on whether the primary field $\left[\phi_{r}\right]$ is allowed or not in the conformal field theory fusion algebra for the primary fields $\left[\phi_{i}\right]$ and $\left[\phi_{j}\right] \quad(i, j, r=0,1 / 2,1, \ldots . k / 2):$

$$
\left[\phi_{i}\right] \otimes\left[\phi_{j}\right]=\sum_{r} N_{i j}^{r}\left[\phi_{r}\right]
$$

Eq. (1) gives the number of conformal blocks with spins $j_{1}, j_{2}, \ldots, j_{p}$ on $p$ external lines and spins $r_{1}, r_{2}, \ldots, r_{p-2}$ on the internal lines. We next take recourse to the Verlinde formula [16]

$$
N_{i j}^{r}=\sum_{s} \frac{S_{i s} S_{j s} S_{s}^{\dagger r}}{S_{0 s}}
$$

where, the unitary matrix $S_{i j}$ diagonalizes the fusion matrix. Upon using the unitarity of the $S$-matrix, the algebra (11) reduces to

$$
N^{\mathcal{P}}=\sum_{r=0}^{k / 2} \frac{S_{j_{1}} S_{j_{2} r} \ldots S_{j_{p} r}}{\left(S_{0 r}\right)^{p-2}}
$$

Now, the matrix elements of $S_{i j}$ are known for the case under consideration $\left(S U(2)_{k}\right.$ WessZumino model); they are given by

$$
S_{i j}=\sqrt{\frac{2}{k+2}} \sin \left(\frac{(2 i+1)(2 j+1) \pi}{k+2}\right),
$$

where, $i, j$ are the spin labels, $i, j=0,1 / 2,1, \ldots k / 2$. Using this $S$-matrix, the number of conformal blocks for the set of punctures $\mathcal{P}$ is given by

\footnotetext{
${ }^{2}$ Similar ideas using self-dual boundary conditions as outer boundary conditions have appeared in 17.
} 


$$
N^{\mathcal{P}}=\frac{2}{k+2} \sum_{r=0}^{k / 2} \frac{\prod_{l=1}^{p} \sin \left(\frac{\left(2 j_{l}+1\right)(2 r+1) \pi}{k+2}\right)}{\left[\sin \left(\frac{(2 r+1) \pi}{k+2}\right)\right]^{p-2}} .
$$

In the notation of [10], eq. (6) gives the dimensionality, dim $\mathcal{H}_{S}^{\mathcal{P}}$, for arbitrary area of the horizon $k$ and arbitrary number of punctures. The dimensionality of the space of states $\mathcal{H}_{\mathcal{S}}$ of CS theory on three-manifold with $S^{2}$ boundary is then given by summing $N^{\mathcal{P}}$ over all sets of punctures $\mathcal{P}: N_{b h}=\sum_{\mathcal{P}} N^{\mathcal{P}}$. Then, the entropy of the black hole is given by $S=\log N_{b h}$

Observe now that eq. (6) can be rewritten, with appropriate redefinition of dummy variables and recognizing that the product can be written as a multiple sum,

$$
N^{\mathcal{P}}=\left(\frac{2}{k+2}\right) \sum_{l=1}^{k+1} \sin ^{2} \theta_{l} \sum_{m_{1}=-j_{1}}^{j_{1}} \cdots \sum_{m_{p}=-j_{p}}^{j_{p}} \exp \left\{2 i\left(\sum_{n=1}^{p} m_{n}\right) \theta_{l}\right\}
$$

where, $\theta_{l} \equiv \pi l /(k+2)$. Expanding the $\sin ^{2} \theta_{l}$ and interchanging the order of the summations, a few manipulations then yield

$$
N^{\mathcal{P}}=\sum_{m_{1}=-j_{1}}^{j_{1}} \cdots \sum_{m_{p}=-j_{p}}^{j_{p}}\left[\delta_{\left(\sum_{n=1}^{p} m_{n}\right), 0}-\frac{1}{2} \delta_{\left(\sum_{n=1}^{p} m_{n}\right), 1}-\frac{1}{2} \delta_{\left(\sum_{n=1}^{p} m_{n}\right),-1}\right],
$$

where, we have used the standard resolution of the periodic Kronecker deltas in terms of exponentials with period $k+2$,

$$
\delta_{\left(\sum_{n=1}^{p} m_{n}\right), m}=\left(\frac{1}{k+2}\right) \sum_{l=0}^{k+1} \exp \left\{2 i\left[\left(\sum_{n=1}^{p} m_{n}\right)-m\right] \theta_{l}\right\}
$$

Notice that the explicit dependence on $k+2$ is no longer present in the exact formula (8).

For large $k$ and large number of punctures $p$ our result (间) reduces to

$$
N^{\mathcal{P}} \sim \prod_{l=1}^{p}\left(2 j_{l}+1\right)
$$

in agreement with the result of ref. [10]. Thus the semiclassical B-H formula is valid in this approximation. To see if the $\mathrm{B}-\mathrm{H}$ formula relating entropy with area is valid even in the quantum theory, one needs to obtain the eigenvalues of the area operator without any assumptions about their size. This might entail a modified regularized operator which 
measures horizon area in the quantum theory and is, in general, a constant of motion, i.e., commutes with the Hamiltonian constraint.

It appears that methods of two dimensional conformal field theory effectively describe quantitative quantum physics of the black holes in four spacetime dimensions. In our work, the conformal field theory enters through the relation the boundary states of the $S U(2)$ Chern-Simons theory have with the conformal blocks of the corresponding conformal field theory. The theory of irreducible representations of the simplest of the conformal field theories, $S U(2)_{k}$ Wess-Zumino model, is crucial to yield what may be thought of as a quantum generalizations of the semiclassical B-H entropy of the black hole. Extensions of our results to the case of charged and rotating black holes will hopefully constitute a future publication, as also attempts to understand Hawking radiation within the canonical quantum gravity approach.

Discussions with S. Carlip, T. R. Govindarajan and C. Rovelli are gratefully acknowledged. 


\section{REFERENCES}

[1] J. Bekenstein, Phys. Rev. D7, 2333 (1973).

[2] S. Hawking, Comm. Math. Phys. 43, 190 (1975).

[3] A. Sen, Mod. Phys. Let. A 10, 2081 (1995), hep-th/9504147.

[4] A. Strominger and C. Vafa, Phys. Lett. B379, 99 (1996), hep-th/9601029.

[5] G. Horowitz, 'Black Hole Entropy from Near-Horizon Microstates', hep-th/9704072.

[6] S. Das and S. Mathur, Nucl. Phys. B478, 561 (1996), hep-th/9606185.

[7] A. Strominger, 'Quantum Black Hole Entropy from Near-Horizon Microstates', hepth/9712252.

[8] A. Ashtekar, Lectures on Non-perturbative Quantum Gravity, (World Scientific, 1991).

[9] K. Krasnov, "On Quantum Statistical Mechanics of a Schwarzschild black hole", gr-qc/9605047, "Quantum geomtetry and thermal radiation from black holes", grqc/9710006.

[10] A. Ashtekar, J. Baez, A. Corichi and K. Krasnov, "Quantum Geometry and Black Hole Entropy", gr-qc/9710007 and references therein.

[11] G. Immirzi, "Quantum Gravity and Regge Calculus", gr-qc/9701052.

[12] A. P. Balachandran. L. Chandar and A. Momen, Nucl. Phys. B461, 581 (1996); S. Carlip, Phys. Rev. D51, 632 (1995).

[13] C. Rovelli and L. Smolin, Phys. Rev. D52, 5743 (1995); S. Fritelli, L. Lehner, C. Rovelli, Class. Quant. Grav. 13, 2921 (1996).

[14] A. Ashtekar and J. Lewandowski, Class. Quant. Grav. 14, 55 (1997) and references therein.

[15] E. Witten, Comm. Math, Phys. 121, 351 (1989). 
[16] P. Di Francesco, P. Mathieu and D. Senechal, Conformal Field Theory, p. 375 et seq (Springer Verlag 1997).

[17] L. Smolin, Jour. Math. Phys. 36, 6417 (1995). 